\title{
Universal star products
}

\author{
Mourad Ammar ${ }^{1,2}$ \\ 1 Université du Luxembourg \\ avenue de la faïencerie \\ mourad.ammar@uni.lu
}

\author{
Véronique Chloup ${ }^{2}$ \\ 2 Université Paul Verlaine - Metz, LMAM \\ Ile du Saulcy, F-57045 Metz Cedex 01,France \\ chloup@poncelet.univ-metz.fr
}

\author{
Simone Gutt ${ }^{3,2}$ \\ Membre de l'Académie Royale de Belgique \\ 3 Université Libre de Bruxelles, Campus Plaine CP 218, \\ Bvd du Triomphe, B-1050 Brussels, Belgium \\ sgutt@ulb.ac.be, gutt@poncelet.univ-metz.fr
}

\begin{abstract}
One defines the notion of universal deformation quantization: given any manifold $M$, any Poisson structure $\Lambda$ on $M$ and any torsionfree linear connection $\nabla$ on $M$, a universal deformation quantization associates to this data a star product on $(M, \Lambda)$ given by a series of bidifferential operators whose corresponding tensors are given by universal polynomial expressions in the Poisson tensor $\Lambda$, the curvature tensor $R$ and their covariant iterated derivatives. Such universal deformation quantization exist. We study their unicity at order 3 in the deformation parameter, computing the appropriate universal Poisson cohomology.
\end{abstract}

Mathematics Subject Classifications (2000): 53D55, 81S10

Keywords: star products, formality, universal differential operators. 


\section{Introduction}

Let $(M, \Lambda)$ be a d-dimensional Poisson manifold. Let $C^{\infty}(M)$ be the commutative algebra of $\mathbb{K}$-valued smooth function on $M$, where $\mathbb{K}$ is $\mathbb{R}$ or $\mathbb{C}$. The Poisson bracket of functions on $(M, \Lambda)$ is denoted by $\{f, g\}:=\Lambda(d f, d g), f, g \in C^{\infty}(M)$, and $m(f, g)=f . g$ denotes the pointwise multiplication.

Definition 1 [1] Let $C^{\infty}(M)[[v]]$ be the space of formal power series in the formal parameter $v$ with coefficients in $C^{\infty}(M)$. A differential star product on $(M, \Lambda)$ is a bilinear map

$$
*: C^{\infty}(M) \times C^{\infty}(M) \rightarrow C^{\infty}(M)[[v]] \quad(f, g) \rightarrow f * g:=f . g+\sum_{r \geq 1} v^{r} C_{r}(f, g),
$$

such that

1. its $\mathbb{K}[[v]]$-bilinear extension is an associative product $(f * g) * h=f *(g * h)$;

2. $C_{1}(f, g)-C_{1}(g, f)=2\{f, g\}$;

3. each $C_{r}$ is a bidifferential operator vanishing on constants.

Kontsevich gave in [10] an explicit formula for a star product on $\mathbb{R}^{d}$ endowed with any Poisson structure, as a special case of his formality theorem for the Hochschild complex of multidifferential operators.

Theorem 1 [10] The formality theorem on $\mathbb{R}^{d}$ is proven through the explicit formula of an $L_{\infty}$ morphism from the differential graded Lie algebra of polyvectorfields on $\mathbb{R}^{d}$ to the differential graded Lie algebra of polydifferential operators on $\mathbb{R}^{d}$. This consists in a collection of multilinear graded symmetric maps $U_{j}$ associating to $j$ multivectorfields $\alpha_{k} \in \Gamma\left(\mathbb{R}^{d}, \wedge^{m_{k}} T \mathbb{R}^{d}\right)$ a multidifferential operator

$$
U_{j}\left(\alpha_{1}, \ldots, \alpha_{j}\right): C^{\infty}\left(\mathbb{R}^{d}\right)^{\otimes r} \rightarrow C^{\infty}\left(\mathbb{R}^{d}\right)
$$

with $r=\sum_{k} m_{k}-2 j+2$, satisfying quadratic relations $(*)$ which translate the fact that they are the Taylor coefficients of a $L_{\infty}$ morphism. The maps $U_{j}$ are defined in terms of graphs; in particular the coefficients of the multidifferential operator $U_{j}\left(\alpha_{1}, \ldots, \alpha_{j}\right)$ are given by multilinear universal expressions in the partial derivatives of the coefficients of the multivectorfields $\alpha_{1}, \ldots, \alpha_{j}$.

The relations $(*)$ imply that, given any Poisson structure $\Lambda$ on $\mathbb{R}^{d}$, the formula

$$
f *_{K}^{\Lambda} g=f g+\sum_{n=1}^{\infty} \frac{v^{n}}{n !} U_{n}(\Lambda, \ldots, \Lambda)(f, g)=f g+v \Lambda(d f, d g)+O\left(v^{2}\right)
$$

defines a star product on $\left(\mathbb{R}^{d}, \Lambda\right)$. Observe that each $U_{n}(\Lambda, \ldots, \Lambda)$ is a bidifferential operator of order maximum $n$ in each argument whose coefficients are polynomials of degree $n$ in the partial derivatives of the coefficients of the tensor $\Lambda$.

Kontsevich then obtained the existence of star products on a general Poisson manifold using abstract arguments.

A more direct construction of a star product on a $d$-dimensional Poisson manifold $(M, P)$, using Kontsevich's formality on $\mathbb{R}^{d}$, was given by Cattaneo, Felder and Tomassini in [3]. Given a torsionfree connexion $\nabla$ on $(M, P)$ one builds an identification of the commutative algebra $C^{\infty}(M)$ of smooth functions on $M$ with the algebra of flat sections of the jet bundle $E \rightarrow M$, for the Grothendieck connection $D^{G}$. The next point is to "quantize" this situation: a deformed algebra stucture on $\Gamma(M, E)[[v]]$ is obtained through fiberwize quantization of the jet bundle using Kontsevich star product on $\mathbb{R}^{d}$, and 
a deformed flat connection $D$ which is a derivation of this deformed algebra structure is constructed "à la Fedosov". Then one constructs an identification between the formal series of functions on $M$ and the algebra of flat sections of this quantized bundle of algebras; this identification defines the star product on $M$.

Later, Dolgushev [7] gave in a similar spirit a construction for a Kontsevich's formality quasiisomorphism for a general smooth manifold. The construction starts again with a torsionfree linear connexion $\nabla$ on $M$ and the identification of the commutative algebra $C^{\infty}(M)$ of smooth functions on $M$ with the algebra of flat sections of the jet bundle $E \rightarrow M$, for a connection $D^{F}$ constructed "à la Fedosov". This is extended to a resolution of the space $T_{\text {poly }}(M)$ of polyvectors on the manifold using the complexes of forms on $M$ with values in the bundle of formal fiberwize polyvectorfields on $E$ and a resolution of the space $D_{\text {poly }}(M)$ of polydifferential operators on the manifold using the complexes of forms on $M$ with values in the bundle of formal fiberwize polydifferential operators on $E$. The fiberwize Kontsevich $L_{\infty}$ morphism is then twisted and contracted to yield a $L_{\infty}$ - morphism from $T_{\text {poly }}(M)$ to $D_{\text {poly }}(M)$.

Given a torsionfree linear connection $\nabla$ on a manifold $M$, any multidifferential operator $\mathrm{Op}$ : $\left(C^{\infty}(M)\right)^{k} \rightarrow C^{\infty}(M)$ writes in a unique way as

$$
\mathrm{Op}\left(f_{1}, \ldots, f_{k}\right)=\sum_{J_{1}, \ldots, J_{k}} \mathrm{Op}^{J_{1}, \ldots, J_{k}} \nabla_{J_{1}}^{s y m} f_{1} \ldots \nabla_{J_{k}}^{\text {sym }} f_{k}
$$

where the $J_{1}, \ldots, J_{k}$ are multiindices and $\nabla_{J}^{s y m} f$ is the symmetrised covariant derivative of order $|J|$ of $f:$

$$
\nabla_{J}^{s y m} f=\sum_{\sigma \in S_{m}} \frac{1}{m !} \nabla_{i_{\sigma(1)} \ldots i_{\sigma(m)}} f \quad \text { for } \mathbf{J}=\left(\mathrm{i}_{1}, \ldots, \mathrm{i}_{\mathrm{m}}\right),
$$

where $\nabla_{i_{1} \ldots i_{m}}^{m} f:=\nabla^{m} f\left(\partial_{i_{1}}, \ldots, \partial_{i_{m}}\right)$ with $\nabla^{m} f$ defined inductively by $\nabla f:=d f$ and $\nabla^{m} f\left(X_{1}, \ldots, X_{m}\right)=\left(\nabla_{X_{1}}\left(\nabla^{m-1} f\right)\right)\left(X_{2}, \ldots, X_{m}\right)$.

The tensors $\mathrm{Op}^{J_{1}, \ldots, J_{k}}$ are covariant tensors of order $\left|J_{1}\right|+\ldots+\left|J_{k}\right|$ which are symmetric within each block of $J_{r}$ indices; they are called the tensors associated to Op for the given connection.

Definition 2 A universal Poisson-related multidifferential operator will be the association to any manifold $M$, any torsionfree connection $\nabla$ on $M$ and any Poisson tensor $P$ on $M$, of a multidifferential operator $\mathrm{Op}^{(M, \nabla, \Lambda)}:\left(C^{\infty}(M)\right)^{k} \rightarrow C^{\infty}(M)$, so that, the tensors associated to $\mathrm{Op}^{(M, \nabla, \Lambda)}$ for $\nabla$ are given by universal polynomials in $\Lambda$, the curvature tensor $R$ and their covariant multiderivatives, involving concatenations.

We shall say that a universal Poisson-related multidifferential operator is of no-loop type if the concatenations only arise between different terms, not within a given term (i.e. $\left(\nabla_{r} \Lambda\right)^{i s}\left(\nabla_{s} \Lambda\right)^{j r} \nabla_{i j}^{s y m}$ is of no-loop type but $\left(\nabla_{t} \Lambda\right)^{t i} \nabla_{i}^{s y m}$ or $R_{s t r}^{r} \Lambda^{s i} \Lambda^{t j} \nabla_{i j}^{s y m}$ are not).

We shall say that the universal Poisson-related operator $\mathrm{Op}^{(M, \nabla, \Lambda)}$ is a polynomial of degree $r$ in the Poisson structure if $\mathrm{Op}^{(M, \nabla, t \Lambda)}=t^{r} \mathrm{Op}^{(M, \nabla, \Lambda)}$.

Definition 3 A universal star product $*=m+\sum_{r \geq 1} v^{r} C_{r}$ will be the association to any manifold $M$, any torsionfree connection $\nabla$ on $M$ and any Poisson tensor $P$ on $M$, of a differential star product $*^{(M, \nabla, \Lambda)}:=m+\sum_{r \geq 1} v^{r} C_{r}^{(M, \nabla, \Lambda)}$ where each $C_{r}$ is a universal Poisson-related bidifferential operator of no-loop type, which is a polynomial of degree $r$ in the Poisson structure.

An example of a universal star product at order 3 is given in section 2 , Unicity at order 3 is studied in section 3 using universal Poisson cohomology. This, we compute for universal Poisson-related bidifferential operators of order 1 in each argument defined by low order 
polynomials in the Poisson structure.

The existence of a universal star product is implied either by the globalisation proof of Cattaneo, Felder and Tomassini [3, 4] using the exponential map of a torsionfree linear connection, either by the globalisation of the formality given by Dolgushev [7]. We show this existence in section 5, stressing first the relations between the resolutions involved in the two constructions in section 4

\section{An example at order 3}

Theorem 2 There exists a universal star product up to order three, which associates to a Poisson manifold $(M, \Lambda)$ and a torsionfree linear connection $\nabla$ on $M$, the star product at order three defined by

$$
f \tilde{\varkappa}_{3}^{(M, \nabla, \Lambda)} g=f . g+v\{f, g\}+v^{2} \tilde{C}_{2}^{(M, \nabla, \Lambda)}(f, g)+v^{3} \tilde{C}_{3}^{(M, \nabla, \Lambda)}(f, g), \quad f, g \in C^{\infty}(M)
$$

for

$$
\tilde{C}_{2}^{(M, \nabla, \Lambda)}(f, g)=\frac{1}{2} \Lambda^{k r} \Lambda^{l s} \nabla_{k l}^{2} f \nabla_{r s}^{2} g+\frac{1}{3} \Lambda^{k r} \nabla_{r} \Lambda^{l s}\left(\nabla_{k l}^{2} f \nabla_{s} g+\nabla_{s} f \nabla_{k l}^{2} g\right)+\frac{1}{6} \nabla_{l} \Lambda^{k r} \nabla_{k} \Lambda^{l s} \nabla_{r} f \nabla_{s} g
$$

and

$$
\tilde{C}_{3}^{(M, \nabla, \Lambda)}(f, g)=\frac{1}{6} S_{\nabla}^{(M, \Lambda) 3}(f, g)=-\frac{1}{6} \Lambda^{l s}\left(\mathscr{L}_{X_{f}} \nabla\right)_{k l}^{j}\left(\mathscr{L}_{X_{g}} \nabla\right)_{j s}^{k} \quad \text { with } X_{f}=i(d f) \Lambda,
$$

where $\mathscr{L}_{X_{f}} \nabla$ is the tensor defined by the Lie derivative of the connection $\nabla$ in the direction of the Hamiltonian vector field $X_{f}$

$$
\left(\mathscr{L}_{X_{f}} \nabla\right)_{k l}^{j}=\Lambda^{i j} \nabla_{k l i}^{3} f+\nabla_{k} \Lambda^{i j} \nabla_{l i}^{2} f+\nabla_{l} \Lambda^{i j} \nabla_{k i}^{2} f+\nabla_{k l}^{2} \Lambda^{i j} \nabla_{i} f+R_{i k l}^{j} \Lambda^{s i} \nabla_{s} f .
$$

This can be seen by direct computation.

Remark 1 The operator $S_{\nabla}^{(M, \Lambda) 3}$ was introduced by Flato, Lichnerowicz and Sternheimer [9]; it is a Chevalley-cocycle on $(M, P)$, i.e.

$$
\underset{u, v, w}{\oplus_{\nabla}}\left\{S_{\nabla}^{(M, \Lambda) 3}(u, v), w\right\}+S_{\nabla}^{(M, \Lambda) 3}(\{u, v\}, w)=0,
$$

where $\underset{u, v, w}{\oplus}$ denotes the sum over cyclic permutations of $u, v, w$.

For this universal star product at order 3, there exists a universal Poisson-related-differential-operatorvalued 1-form $D$ defined as follows:

Proposition 1 Given any Poisson manifold $(M, \Lambda)$, any torsionfree linear connection $\nabla$ on $M$, and any vector field $X$ on $M$, the differential operator $D_{X}^{(M, \nabla, \Lambda)}$ defined by

$$
D_{X}^{(M, \nabla, \Lambda)} g=X g-v^{2} \frac{1}{6} \Lambda^{l s}\left(\mathscr{L}_{X} \nabla\right)_{k l}^{j}\left(\mathscr{L}_{X_{g}} \nabla\right)_{j s}^{k}, \quad g \in C^{\infty}(M)
$$

verifies at order 3 in $v$

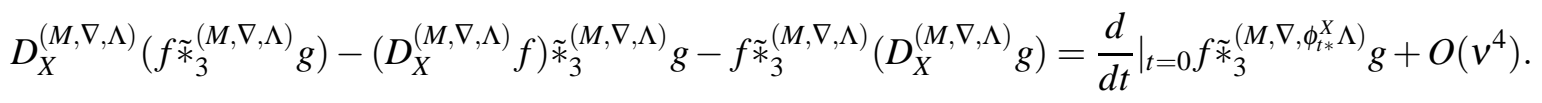


where $\phi_{t}^{X}$ denotes the flow of the vectorfield $X$.

If $X$ is a Hamiltonian vector field corresponding to a function $f \in C^{\infty}(M)$, then $D_{X_{f}}^{(M, \nabla, \Lambda)}$ coincides with the inner derivation at order 3 of $\tilde{*}_{3}$ defined by the function $f$, i.e.

$$
D_{X_{f}}^{(M, \nabla, \Lambda)} g=\frac{1}{2 v}\left(f \widetilde{*}_{3}^{(M, \nabla, \Lambda)} g-g \widetilde{*}_{3}^{(M, \nabla, \Lambda)} f\right) .
$$

\section{Equivalence of universal star products - Universal Poisson cohomol- ogy}

Lemma 1 - Any universal star product $*=m+\sum_{r \geq 1} v^{r} C_{r}$ is a natural star product, i.e. each bidifferential operator $C_{r}$ is of order at most $r$ in each argument. Indeed $C_{r}$ is a universal Poisson-related bidifferential operator defined by a polynomial of degree $r$ in the Poisson structure; this implies, in view of the Bianchi's identities for the curvature tensor, that $C_{r}$ is of order at most $r$ in each argument.

- The universal Poisson-related bidifferential operator $C_{1}$ of any universal star product is necessarily the Poisson bracket $C_{1}^{(M, \nabla, \Lambda)}=\Lambda^{i j} \nabla_{i} \wedge \nabla_{j}$.

- The Gerstenhaber bracket $[,]_{G}$ of two universal Poisson-related multidifferential operator of degree $k$ and $l$ in $\Lambda$, is a universal Poisson-related multidifferential operator of degree $k+l$ in $\Lambda$.

- If a universal Poisson-related p-differential operator $C$ is a Hochschild p-cocycle ( where $\partial:=\operatorname{ad} m=[m, .]_{G}$ denotes the Hochschild differential) then $C=A+\partial B$ where A a universal Poisson-related p-differential operator which is of order 1 in each argument and is the totally skewsymmetric part of $C$, and where $B$ is a universal Poisson-related ( $p-1)$-differential operator.

The last point comes from the explicit formulas [2, 5] for the tensors associated to $B$ in terms of those associated to $C$ when one is given a connection.

Definition 4 A universal Poisson $p$-cocycle is a universal Poisson-related p-differential skewsymmetric operator $C$ of order 1 in each argument which is a cocycle for the Chevalley cohomology for the adjoint representation of $\left(C^{\infty}(M),\{\},\right)$, i.e. with the coboundary defined by

$$
\begin{aligned}
\delta_{P} C\left(u_{1}, \ldots, u_{m+1}\right)= & \sum_{i=1}^{m+1}(-1)^{i}\left\{u_{i}, C\left(u_{1}, \ldots \hat{u}_{i} \ldots, u_{m+1}\right)\right\} \\
& +\sum_{i<j}(-1)^{i+j} C\left(\left\{u_{i}, u_{j}\right\}, u_{1} \ldots \hat{u}_{i} \ldots \hat{u}_{j} \ldots, u_{m+1}\right) .
\end{aligned}
$$

which can be written as a multiple of

$$
\operatorname{skew}\left[\Lambda, C^{(M, \nabla, \Lambda)}\right]_{G}
$$

where skew indicates the skewsymmetrisation in all its arguments of an operator.

Equivalently, a universal Poisson $p$-cocycle $C$ is defined by a universal Poisson related skewsymmetric $p$-tensor $c$ (with $\left.C\left(u_{1}, \ldots, u_{p}\right)=c\left(d u_{1}, \ldots, d u_{p}\right)\right)$ so that

$$
\left[\Lambda, c^{(M, \nabla, \Lambda)}\right]_{S N}=0
$$


where $[\cdot, \cdot]_{S N}$ denotes the Schouten-Nijenhuis bracket of skewsymmetric tensors (which is the extension, as a graded derivation for the exterior product $\wedge$ of the usual bracket of vectorfields).

A universal Poisson $p$-cocycle $C$ is a universal Poisson coboundary if there exists a universal Poisson-related skewsymmetric $(p-1)$-differential operator $C$ of order 1 in each argument so that

$$
C^{(M, \nabla, \Lambda)}=\delta_{P} B^{(M, \nabla, \Lambda)}\left(=\operatorname{skew}\left[\Lambda, B^{(M, \nabla, \Lambda)}\right]_{G}\right) ;
$$

(equivalently, if there exists a universal Poisson related tensor $b$ so that $\left.c^{(M, \nabla, \Lambda)}=\left[\Lambda, b^{(M, \nabla, \Lambda)}\right]_{S N}\right)$.

The universal Poisson cohomology $H^{p}$ is the quotient of the space of universal Poisson $p$-cocycles by the space of universal Poisson $p$-coboundaries.

We can restrict ourselves to the space of universal Poisson $p$-cocycles defined by polynomials of degree $k$ in the Poisson structures and make the quotient by the space of universal Poisson coboundaries defined by polynomials of degree $k-1$. We speak then of the universal Poisson $p$-cohomology of degree $k$ in the Poisson structure and we denote it by $H_{p o l k}^{p}$. We can further restrict ourselves to universal Poisson related tensors (or operators of order 1 in each argument) of no-loop type.

Definition 5 If $*=m+\sum_{r \geq 1} v^{r} C_{r}$ is a universal star product and if $E=\sum_{r=2}^{\infty} v E_{r}$ is a formal series of universal differential operators vanishing on constants, of no-loop type, with each $E_{r}$ a polynomial of degree $r$ in the Poisson structure, then the series $*^{\prime}$ defined by

$$
*^{\prime}=(\exp \operatorname{ad} E) *
$$

where $\operatorname{ad} E \cdot=[E, \cdot]_{G}$, (i.e. $\left.f *^{\prime} g=\exp E((\exp -E) f *(\exp -E) g)\right)$, is an equivalent universal star product. We say that $*$ and $*^{\prime}$ are universally equivalent.

Lemma 2 If $*$ and $*^{\prime}$ are universal star products which coincide at order $k$ in the deformation parameter $v$, then, by the associativity relation at order $k, C_{k}^{\prime}-C_{k}$ is a universal Hochschild 2-cocycle of no-loop type which is a polynomial of degree $k$ in the Poisson structure. Furthermore, associativity at order $k+1$ implies that its skewsymmetric part $p_{2}$ is a universal Poisson 2-cocycle :

$$
\underset{u, v, w}{\varpi}\left\{p_{2}^{M, \nabla, \Lambda}(u, v), w\right\}+p_{2}^{M, \nabla, \Lambda}(\{u, v\}, w)=0,
$$

where $\underset{u, v, w}{\uplus}$ denotes the sum over cyclic permutations of $u, v, w$.

If it is a universal Poisson 2-coboundary of no-loop type, then there is a a formal series $E$ of universal differential operators vanishing on constants such that $(\exp \operatorname{ad} E) *$ and $*^{\prime}$ coincide at order $k+1$.

In particular, two universal star products are universally equivalent if $H_{(\text {no-loop,pol })}^{2}=\{0\}$. They are always equivalent at order $k$ in the deformation parameter $v$ if $H_{(\text {no-loop }) \text { pol } j}^{2}=\{0\} \forall 1 \leq j \leq k$.

Consider now any universal star product $*=m+\sum_{r \geq 1} v^{r} C_{r}$. We automatically have that $C_{1}$ is the Poisson bracket. Associativity at order 2 yields $\partial C_{2}=\partial \tilde{C}_{2}^{(M, \nabla, \Lambda)}$ so

$$
C_{2}(f, g)=\tilde{C}_{2}^{(M, \nabla, \Lambda)}(f, g)+p_{2}(f, g)+\partial E_{2}(f, g)
$$

and the skewsymmetric part of associativity at order 3 yields that $p_{2}$ is a universal Poisson 2-cocycle (which is a polynomial of degree 2 in the Poisson structure).

Proposition 2 The spaces $H_{\text {pol2 }}^{2}(\Lambda)$ and $H_{(n o-l o o p) \text { pol } 2}^{2}(\Lambda)$ of universal Poisson 2-cohomology of degree 2 in the Poisson structure vanish. 
ProOF The universal skewsymmetric 2-tensors of degree 2 in $\Lambda$ are combinations of

$$
\begin{aligned}
& \nabla_{s} \Lambda^{i r} \nabla_{r} \Lambda^{j s} \nabla_{i} \wedge \nabla_{j}, \\
& \left(\nabla_{r s}^{2} \Lambda^{i r} \Lambda^{j s}-\nabla_{r s}^{2} \Lambda^{j r} \Lambda^{i s}\right) \nabla_{i} \wedge \nabla_{j}, \\
& \left(\Lambda^{i r} \Lambda^{s t} R_{r s t}^{j}-\Lambda^{j r} \Lambda^{s t} R_{r s t}^{i}\right) \nabla_{i} \wedge \nabla_{j} \\
& \Lambda^{r i} \Lambda^{s j} R_{r s t}^{t} \nabla_{i} \wedge \nabla_{j}
\end{aligned}
$$

The only universal cocycles are the multiples of

$$
\Lambda^{r i} \Lambda^{s j} R_{r s t}^{t} \nabla_{i} \wedge \nabla_{j}
$$

and those are the boundaries of the multiples of $\nabla_{r} \Lambda^{i r} \partial_{i}$. Remark that there are no cocycles of no-loop type.

Thus, universal star product at order 2 are unique modulo equivalence and one can assume that $C_{2}=\tilde{C}_{2}^{(M, \nabla, \Lambda)}$. Then the skewsymmetric part of the Hochschild 2-cocycle $C_{3}-\tilde{C}_{3}^{(M, \nabla, \Lambda)}$ is a universal Poisson 2-cocycle of no-loop type which is a polynomial of degree 3 in the Poisson structure.

Proposition 3 The space $H_{(\text {no-loop }) \text { pol3 }}^{2}(\Lambda)$ of universal Poisson 2-cohomology of no-loop type and of degree 3 in the Poisson structure vanishes.

ProOF We consider all possible universal Poisson 2-cochains of no loop type which are polynomials of degree 3 in the Poisson structure. They are defined by universal skewsymmetric 2-tensors of degree 3 in $\Lambda$ which are combinations with constant coefficients of the different concatenations (with no loops) of

$$
\begin{aligned}
& \Lambda^{*} \Lambda^{*} \Lambda^{*}\left(\nabla_{. .}^{2} R\right)_{\ldots}^{\cdot} \quad \Lambda^{*} \Lambda^{*} \Lambda^{*} R_{\ldots} R_{\ldots}, \\
& \Lambda^{*}(\nabla . \Lambda)^{*}(\nabla . \Lambda)^{*} R_{\ldots} . \quad \Lambda^{*} \Lambda^{*}(\nabla . \Lambda)^{*}(\nabla . R)^{\prime}
\end{aligned}
$$

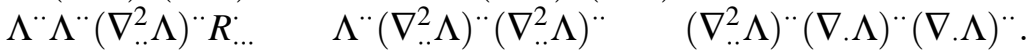

Using the symmetry properties of $R$, the Bianchi's identities and the fact that $\Lambda$ is a Poisson tensor, one is left with a combination with constant coefficients of 49 independant terms.

Universal 2-coboundaries come from the boundaries of universal 1-tensors of degree 2 in $\Lambda$; such 1-tensors are given by combinations with constant coefficients of concatenations of

$$
\Lambda^{*} \Lambda^{*}(\nabla . R)_{\ldots}^{*} \quad \Lambda^{*}(\nabla . \Lambda)^{*} R_{\ldots} .
$$

Hence, modulo universal coboundaries, one can assume that the coefficients of 4 of the 49 terms in a universal cochain are zero.

The cohomology that we are looking for is then given by the combinations with constant coefficients of the remaining 45 terms which are cocycles (for all possible choices of manifold, Poisson structure $\Lambda$ and connection $\nabla$.)

Let $C$ be a combination of those 45 terms. The cocycle condition is $[\Lambda, C]_{S N}=0$. We plug in examples of Poisson structures and connections and impose this cocycle condition. This shows that all 45 coefficients must vanish. 
It is enough, for instance, to consider the example on $\mathbb{R}^{4}$, with the non vanishing coefficients of the connection defined by

$$
\begin{aligned}
& \Gamma_{12}^{1}=x_{1}^{3}, \quad \Gamma_{14}^{1}=x_{4} \quad \Gamma_{11}^{2}=x_{1}^{2}, \quad \Gamma_{13}^{2}=1, \quad \Gamma_{22}^{2}=1, \quad \Gamma_{14}^{2}=x_{3}, \quad \Gamma_{13}^{3}=-x_{4}, \\
& \Gamma_{33}^{3}=1, \quad \Gamma_{44}^{3}=-x_{2} x_{3} x_{4}, \quad \Gamma_{11}^{4}=1, \quad \Gamma_{13}^{4}=1, \quad \Gamma_{22}^{4}=x_{1}, \quad \Gamma_{44}^{3}=-3 .
\end{aligned}
$$

and the quadratic Poisson structure defined by

$$
\Lambda=\sum_{1=i<j=4} x_{i} x_{j} \frac{\partial}{\partial x_{i}} \wedge \frac{\partial}{\partial x_{j}}
$$

From this example, one gets that 41 of the 45 coefficients have to vanish. One is left with a combination with constant coefficients of four terms and an example with constant Poisson structure in dimension 7 shows that all those coefficients must vanish. The non vanishing coefficients of this example are:

$$
\begin{aligned}
& \Lambda^{12}=1 \quad \Lambda^{15}=1 \quad \Lambda^{17}=1 \quad \Lambda^{25}=1 \quad \Lambda^{26}=2 \quad \Lambda^{27}=2 \\
& \Lambda^{34}=1 \quad \Lambda^{37}=3 \quad \Lambda^{46}=1 \quad \Lambda^{47}=4 \quad \Lambda^{56}=1 \quad \Lambda^{57}=5 \quad \Lambda^{67}=6 . \\
& \Gamma_{12}^{1}=1 \quad \Gamma_{62}^{1}=x_{7} \quad \Gamma_{77}^{1}=-1 \quad \Gamma_{17}^{2}=x_{1} \quad \Gamma_{13}^{3}=x_{6} \quad \Gamma_{11}^{4}=1 \quad \Gamma_{22}^{4}=1 \quad \Gamma_{33}^{4}=2 \\
& \Gamma_{77}^{4}=3 \quad \Gamma_{12}^{5}=x_{1} x_{5} \quad \Gamma_{33}^{5}=x_{2} \quad \Gamma_{11}^{6}=1 \quad \Gamma_{44}^{6}=x_{2} \quad \Gamma_{44}^{6}=x_{5} \quad \Gamma_{11}^{7}=x_{7} \quad \Gamma_{44}^{7}=x_{3} \quad \Gamma_{17}^{7}=x_{1} .
\end{aligned}
$$

Corollary 1 Any universal star product is universally equivalent to one whose expression at order 3 is given by formula (2.1).

\section{Grothendieck- and Dolgushev-resolution of the space of functions}

Our purpose in this section is to prove that the Fedosov-resolution of the algebra of smooth functions constructed in Dolgushev [7] coincides with its resolution given by Cattaneo, Felder and Tomassini in [3]. We also give explicitely the identification of smooth functions, tensorfields and differential operators on $M$ with flat sections in the corresponding bundles.

Let $M$ be a $d$-dimensional manifold and consider the jet bundle $E \rightarrow M$ (the bundle of infinite jet of functions) with fibers $\mathbb{R}\left[\left[y^{1}, \ldots, y^{d}\right]\right]$ (i.e. formal power series in $y \in \mathbb{R}^{d}$ with real coefficients) and transition functions induced from the transition functions of the tangent bundle $T M$. Thus

$$
E=F(M) \times_{\mathrm{Gl}(d, \mathbb{R})} \mathbb{R}\left[\left[y^{1}, \ldots, y^{d}\right]\right]
$$

where $F(M)$ is the frame bundle. Remark that $E$ can be seen as the formally completed symmetric algebra of the cotangent bundle $T^{*} M$; a section $s \in \Gamma(M, E)$ can be written in the form

$$
s=s(x ; y)=\sum_{p=0}^{\infty} s_{i_{1} \ldots i_{p}}(x) y^{i_{1}} \cdots y^{i_{p}}
$$

with repeated indices variing from 1 to $d$, and where the $s_{i_{1} \ldots i_{p}}$ are components of symmetric covariant tensors on $M$. This bundle $E$ is denoted $\mathscr{S} M$ by Dolgushev.

The construction of a star product on a $d$-dimensional Poisson manifold $(M, P)$ given by Cattaneo, Felder and Tomassini in [3], using a linear torsionfree connection $\nabla$ on the manifold $M$, starts 
with the identification of the commutative algebra $C^{\infty}(M)$ of smooth functions on $M$ with the algebra $\mathscr{Z}^{0}\left(\Gamma(M, E), D^{G}\right)$ of flat sections of the jet bundle $E \rightarrow M$, for the Grothendieck connection $D^{G}$ (which is constructed using $\nabla$ ). Let us recall this construction.

The exponential map for the connection $\nabla$ gives an identification

$$
\exp _{x}: U \cap T_{x} M \rightarrow M \quad y \mapsto \exp _{x}(y)
$$

at each point $x$, of the intersection of the tangent space $T_{x} M$ with a neighborhood $U$ of the zero section of the tangent bundle $T M$ with a neighborhood of $x$ in $M$.

To a function $f \in C^{\infty}(M)$, one associates the section $f_{\phi}$ of the jet bundle $E \rightarrow M$ given, for any $x \in M$ by the Taylor expansion at $0 \in T_{x} M$ of the pullback $f \circ \exp _{x}$.

Lemma 3 The section $f_{\phi}$ is given by:

$$
f_{\phi}(x ; y)=f(x)+\sum_{n>0} \frac{1}{n !} \nabla_{i_{1} \ldots i_{n}}^{n} f(x) y^{i_{1}} \ldots y^{i_{n}}=f(x)+\sum_{n>0} \frac{1}{n !} \nabla_{i_{1} \ldots i_{n}}^{n, s y m} f(x) y^{i_{1}} \ldots y^{i_{n}} .
$$

PROOF In local coordinates $x^{i}$ 's one has $\frac{d}{d t} f\left(\exp _{x} t y\right)=\sum_{k=1}^{d}\left(\partial_{x^{k}} f\right)\left(\exp _{x} t y\right) \frac{d}{d t}\left(\exp _{x} t y\right)^{k}$ and $\frac{d^{2}}{d t^{2}} f\left(\exp _{x} t y\right)=\sum_{k, l}\left(\partial_{x^{k} x^{l}}^{2} f\right)\left(\exp _{x} t y\right) \frac{d}{d t}\left(\exp _{x} t y\right)^{k} \frac{d}{d t}\left(\exp _{x} t y\right)^{l}+\sum_{k=1}^{d}\left(\partial_{x^{k}} f\right)\left(\exp _{x} t y\right) \frac{d^{2}}{d t^{2}}\left(\exp _{x} t y\right)^{k}$. The definition of the exponential map imply that

$$
\frac{d^{2}}{d t^{2}}\left(\exp _{x} t y\right)^{k}=-\sum_{r, s} \Gamma_{r s}^{k}\left(\exp _{x} t y\right) \frac{d}{d t}\left(\exp _{x} t y\right)^{r} \frac{d}{d t}\left(\exp _{x} t y\right)^{s}
$$

hence $\frac{d^{2}}{d t^{2}} f\left(\exp _{x} t y\right)=\sum_{k, l}\left(\nabla_{k l}^{2} f\right)\left(\exp _{x} t y\right) \frac{d}{d t}\left(\exp _{x} t y\right)^{k} \frac{d}{d t}\left(\exp _{x} t y\right)^{l}$. By induction, one gets

$$
\frac{d^{n}}{d t^{n}} f\left(\exp _{x} t y\right)=\sum_{k_{1}, \ldots k_{n}}\left(\nabla_{k_{1} \ldots k_{n}}^{n} f\right)\left(\exp _{x} t y\right) \frac{d}{d t}\left(\exp _{x} t y\right)^{k_{1}} \ldots \frac{d}{d t}\left(\exp _{x} t y\right)^{k_{n}}
$$

and the result follows at $t=0$.

Definition 6 [4] The Grothendieck connection $D^{G}$ on $E$ is defined by:

$$
D_{X}^{G} s(x ; y):=\left.\frac{d}{d t}\right|_{t=0} s\left(x(t) ; \exp _{x(t)}^{-1}\left(\exp _{x}(y)\right)\right)
$$

for any curve $t \rightarrow x(t) \in M$ representing $X \in T_{x} M$ and for any $s \in \Gamma(M, E)$. It is locally given by

$$
D_{X}^{G}=\sum_{i=1}^{d} X^{i}\left(\partial_{x^{i}}+\sum_{k} \sum_{j}\left(\frac{\partial \phi_{x}}{\partial y}\right)_{j}^{k} \frac{\partial \phi^{j}}{\partial x^{i}} \partial_{y^{k}}\right)
$$

where $\phi_{x}(y)=\phi(x, y)$ is the Taylor expansion of $\exp _{x} y$ at $y=0$ :

$$
\phi(x, y)^{k}=x^{k}+y^{k}-\frac{1}{2} \sum_{r s} \Gamma_{r s}^{k}(x) y^{r} y^{s}+\frac{1}{3 !} \sum_{r s t}\left(-\left(\partial_{x^{r}} \Gamma_{s t}^{k}\right)(x)+2 \sum_{u} \Gamma_{r s}^{u}(x) \Gamma_{u t}^{k}(x)\right) y^{r} y^{s} y^{t}+O\left(y^{4}\right) .
$$


Remark $2 \bullet$ From the definition (4.5) it is clear that $D^{G}$ is flat $\left(D_{X}^{G} \circ D_{Y}^{G}-D_{Y}^{G} \circ D_{X}^{G}=D_{[X, Y]}^{G}\right)$.

- It is also obvious that $D^{G}\left(f_{\phi}\right)=0 \forall f \in C^{\infty}(M)$.

Lemma 4 [4] Introducing the operator on E-valued forms on $M$

$$
\delta=\sum_{i} d x^{i} \frac{\partial}{\partial y^{i}}
$$

one can write

$$
D^{G}=-\delta+\nabla^{\prime}+A
$$

where

$$
\nabla^{\prime}=\sum_{i} d x^{i}\left(\partial_{x^{i}}-\sum_{j k} \Gamma_{i j}^{k} y^{j} \partial_{y^{k}}\right)
$$

is the covariant derivative on $E$ associated to $\nabla$ and where $A$ is a 1-form on $M$ with values in the fiberwize vectorfields on $E$,

$$
A(x ; y)=: \sum_{i k} d x^{i} A_{i}^{k}(x ; y) \partial_{y^{k}}=\sum_{i k} d x^{i}\left(-\frac{1}{3} \sum_{r s} R_{r i s}^{k}(x) y^{r} y^{s}+0\left(y^{3}\right)\right) \partial_{y^{k}} .
$$

One extends as usual the operator $D^{G}$ to the space $\Omega(M, E)$ of $E$-valued forms on $M$ :

$$
D^{G}=-\delta+\nabla^{\prime}+A \text { with } \nabla^{\prime}=d-\sum_{i j k} d x^{i} \Gamma_{i j}^{k} y^{j} \partial_{y^{k}}
$$

One introduces the operator $\delta^{*}=\sum_{j} y^{j} i\left(\frac{\partial}{\partial x^{j}}\right)$ on $\Omega(M, E)$. Clearly $\left(\delta^{*}\right)^{2}=0, \delta^{2}=0$ and for any $\omega \in \Omega^{q}\left(M, E_{p}\right)$, i.e. a $q$-form of degree $p$ in $y$, we have $\left(\delta \delta^{*}+\delta^{*} \delta\right) \omega=(p+q) \omega$.

Defining, for any $\omega \in \Omega^{q}\left(M, E_{p}\right)$

$$
\begin{aligned}
\delta^{-1} \omega & =\frac{1}{p+q} \delta^{*} \omega & & \text { when } p+q \neq 0 \\
& =0 & & \text { when } p=q=0
\end{aligned}
$$

we see that any $\delta$-closed $q$-form $\omega$ of degree $p$ in $y$, when $p+q>0$, writes uniquely as $\omega=\delta \sigma$ with $\delta^{*} \sigma=0 ; \sigma$ is given by $\sigma=\delta^{-1} \omega$.

One proceeds by induction on the degree in $y$ to see that the cohomoly of $D^{G}$ is concentrated in degree 0 and that any flat section of $E$ is determined by its part of degree 0 in $y$. Indeed a $q$-form $\omega$ is $D^{G_{-}}$ closed iff $\delta \omega=\left(\nabla^{\prime}+A\right) \omega$; this implies that $\delta \omega_{p}=0$ for $\omega_{p}$ the terms of lowest order $(p)$ in $y$. When $p+q>0$ we can write $\omega_{p}=\delta\left(\delta^{-1} \omega_{p}\right)$ and $\omega-D^{G}\left(\delta^{-1} \omega_{p}\right)$ has terms of lowest order at least $p+1$ in $y$. Remark that given any section $s$ of $E$ then $s(x ; y=0)$ determines a smooth function $f$ on $M$. If $D^{G} s=0$, then $s-f_{\phi}$ is still $D^{G}$ closed. By the above, Its terms of lowest order in $y$ must be of the form $\delta \sigma$ hence must vanish since we have a 0 -form. Hence we have:

Lemma 5 [4] Any section of the jet bundle $s \in \Gamma(E)$ is the Taylor expansion of the pullback of a smooth function $f$ on $M$ via the exponential map of the connection $\nabla$ if and only if it is horizontal for the Grothendieck-connection $D^{G}$ :

$$
s=f_{\phi} \text { for a } f \in C^{\infty}(M) \Leftrightarrow s \in \Gamma_{h o r}(E):=\left\{s^{\prime} \in \Gamma(E) \mid D^{G} s^{\prime}=0\right\} .
$$

Furthermore, the cohomology of $D^{G}$ is concentrated in degree 0 . In other word, one obtains a "Grothendieck-resolution" of the algebra of smooth functions, i.e.

$$
H^{\bullet}\left(\Omega(M, E), D^{G}\right)=H^{0}\left(\Omega(M, E), D^{G}\right)=\Gamma_{h o r}(E) \cong C^{\infty}(M)
$$


Remark 3 When a $q$-form $\omega$ is $D^{G}$ exact, we have written $\omega=D^{G} \sigma$ where the tensors defining $\sigma$ are given by universal polynomials (with no-loop concatenations) in the tensors defining $\omega$, the tensors defining $A$, the curvature of the connection, and their iterated covariant derivatives.

Lemma 6 The 1-form $A$ on $M$ with values in the fiberwize vectorfields on $E$ is given by $A(x ; y)=$ : $\sum_{i k} d x^{i} A_{i}^{k}(x ; y) \partial_{y_{k}}$ where the $A_{i}^{k}$ are universal polynomials given by (no-loop) concatenations of iterative covariant derivatives of the curvature; they are of the form

$$
\sum\left(\nabla_{\ldots} R\right)_{i . .}^{j_{1}}\left(\nabla_{\ldots} R\right)_{j_{1} \ldots}^{j_{2}} \ldots\left(\nabla_{\ldots} R\right)_{j_{s-1} . .}^{k} y^{\prime} \ldots y^{\prime}
$$

In particular $\delta^{-1} A=0$ since the curvature is skewsymmetric in its first two lower arguments. The 1-form $A$ is uniquely characterized by the fact that $\delta^{-1} A=0$. and the fact that $D^{G}=-\delta+\nabla^{\prime}+A$ is flat, i.e; $\left(D^{G}\right)^{2}=0$ which is equivalent to

$$
\delta A=R^{\nabla^{\prime}}+\nabla^{\prime} A+\frac{1}{2}[A, A]
$$

for $\frac{1}{2}[A, A](X, Y):=[A(X), A(Y)]$ and $R^{\nabla^{\prime}}=-\frac{1}{2} R_{i j k}^{l} d x^{i} \wedge d x^{j} y^{k} \frac{\partial}{\partial y^{\prime}}$.

ProOF Any section $s \in \Gamma(M, E)$ writes $\sum_{p=0}^{\infty} s_{i_{1} \ldots i_{p}}^{p}(x) y^{i_{1}} \cdots y^{i_{p}}$ with symmetric $p$-covariant tensors $s_{i_{1} \ldots i_{p}}^{p}$. Write $A(x ; y)=\sum_{r \geq 2} d x^{i}\left(A^{(r)}(x)\right)_{i, j_{1} \ldots j_{r}}^{k} y^{j_{1}} \ldots y^{j_{r}} \partial_{y^{k}}$ with $\left(A^{(2)}(x)\right)_{i, r s}^{k}=-\frac{1}{3} \sum_{r s} R_{r i s}^{k}(x) y^{r} y^{s}$. Then the covariant tensors of $D_{X}^{G}$ are given by the symmetrisation of

$$
\left(D_{X}^{G} s\right)^{p}=-i(X) s^{p+1}+\nabla_{X} s^{p}+\sum_{r=0}^{p-2}\left(A^{(p-r)}(X)\right)^{k} \partial_{y^{k}} s^{r+1} .
$$

The fact that $D^{G}\left(f_{\phi}\right)=0 \forall f \in C^{\infty}(M)$ implies the expression given in the lemma for $A$. Indeed, the symmetric tensors defining $f_{\phi}$ are given by $\frac{1}{p !} \nabla^{p, s y m} f$ and we must have

$$
\begin{gathered}
0=\left(D_{\partial_{x_{i}}}^{G} f_{\phi}\right)_{j_{1} \ldots j_{p}}^{p}=-\frac{1}{(p+1) !}\left(\nabla^{p+1, s y m} f\right)_{i j_{1} \ldots j_{p}}+\frac{1}{p !}\left(\nabla\left(\nabla^{p, s y m} f\right)\right)_{i j_{1} \ldots j_{p}} \\
+\sum_{r=0}^{p-2}\left(A^{(p-r)}(x)\right)_{i, j_{1} \ldots j_{p-r}}^{k} \frac{1}{r !}\left(\nabla^{r+1, s y m} f\right)_{k j_{p-r+1} \ldots j_{p}}
\end{gathered}
$$

with the last terms symmetrized in the $j^{\prime} s$. The commutation of covariant derivatives of a $q$-form $\omega$ gives

$$
\left(\nabla^{p+2} f\right)_{k l j_{1} \ldots j_{p}}-\left(\nabla^{p+2} f\right)_{l k j_{1} \ldots j_{p}}=-\sum_{r=0}^{p} R_{k l j_{r}}^{s}\left(\nabla^{p} f\right)_{j_{1} \ldots j_{r-1} s j_{r+1} \ldots j_{p}}
$$

and implies by induction that $\left(\nabla\left(\nabla^{p, s y m} f\right)\right)_{i j_{1} \ldots j_{p}}-\left(\nabla^{p+1, s y m} f\right)_{i j_{1} \ldots j_{p}}$ is a universal expression contracting covariant derivatives of the curvature tensor with lower covariant derivatives of $f$ of the form

$$
\left(\nabla_{\ldots} R\right)_{i . .}^{t_{1}}\left(\nabla_{\ldots} R\right)_{t_{1} \ldots}^{t_{2}} \ldots\left(\nabla_{\ldots} R\right)_{t_{s-1} . .}^{s}\left(\nabla^{r+1, s y m} f\right)_{s \ldots}
$$

with the $j^{\prime} s$ put in a symmetrised way at the 's, and for $0 \leq r \leq p-2$. Hence the expression for $A$.

Observe that $d \delta+\delta d=0$ and also $\delta \nabla^{\prime}+\nabla^{\prime} \delta=0$ since $\nabla$ is torsionfree. Hence $\left(D^{G}\right)^{2}=0$ iff $-\delta A+R^{\nabla^{\prime}}+\nabla^{\prime} A+\frac{1}{2}[A, A]$ vanishes on all sections of $E$; since it is a 2 -form on $M$ with values in the fiberwize vectorfields on $E$, this must vanish. 
Dolgushev [7] gave in a similar spirit a construction for a Kontsevich's formality quasi-isomorphism for a general smooth manifold. The construction starts again with a torsionfree linear connexion $\nabla$ on $M$. A resolution (called Fedosov's resolution in Dolgushev's paper) of the algebra of functions is given using the complex of algebras $\left(\Omega(M, E), D_{F}\right)$ for a flat connexion (differential) $D_{F}$ defined by

$$
D_{F}:=\nabla^{\prime}-\delta+A
$$

where $A$ is a 1-form on $M$ with values in the fiberwize vectorfields on $E$, obtained by induction on the order in $y$ by the equation

$$
A=\delta^{-1} R^{\nabla^{\prime}}+\delta^{-1}\left(\nabla^{\prime} A+\frac{1}{2}[A, A]\right) .
$$

This implies that $\delta^{-1} A=0$ and $\delta A=R^{\nabla^{\prime}}+\nabla^{\prime} A+\frac{1}{2}[A, A]$ so that $A$ coincides with the 1 -form already considered. Hence

Lemma 7 The differential $D^{G}$ and $D_{F}$ coincide.

Similarly, Dolgushev defined a resolution of polydifferential operators and polyvectorfields on $M$ using the complexes $\left(\Omega\left(M, \mathscr{D}_{\text {poly }}\right), D_{F}^{\mathscr{D}_{\text {poly }}}\right)$ and $\left(\Omega\left(M, \mathscr{T}_{\text {poly }}\right), D_{F}^{\mathscr{T}_{\text {poly }}}\right)$ where $\mathscr{T}_{\text {poly }}$ is the bundle of formal fiberwize polyvectorfields on $E$ and $\mathscr{D}_{\text {poly }}$ is the bundle of formal fiberwize polydifferential operators on $E$. A section of $\mathscr{T}_{\text {poly }}^{k}$ is of the form

$$
\mathscr{F}(x ; y)=\sum_{n=0}^{\infty} \mathscr{F}_{i_{1} \ldots i_{n}}^{j_{1} \ldots j_{k+1}}(x) y^{i_{1}} \ldots y^{i_{n}} \frac{\partial}{\partial y^{j_{1}}} \wedge \ldots \wedge \frac{\partial}{\partial y^{j_{k+1}}},
$$

where $\mathscr{F}_{i_{1} \ldots i_{n}}^{j_{1} \ldots j_{k+1}}(x)$ are coefficients of tensors, symmetric in the covariant indices $i_{1}, \ldots, i_{n}$ and antisymmetric in the contravariant indices $j_{1}, \ldots, j_{k+1}$. A section of $\mathscr{D}_{\text {poly }}^{k}$ is of the form

$$
\mathscr{O}(x ; y)=\sum_{n=0}^{\infty} \mathscr{O}_{i_{1} \ldots i_{n}}^{\alpha_{1} \ldots \alpha_{k+1}}(x) y^{i_{1}} \ldots y^{i_{n}} \frac{\partial^{\left|\alpha_{1}\right|}}{\partial y^{\alpha_{1}}} \otimes \ldots \otimes \frac{\partial^{\left|\alpha_{k+1}\right|}}{\partial y^{\alpha_{k+1}}}
$$

where the $\alpha_{l}$ are multi-indices and $\mathscr{O}_{i_{1} \ldots i_{n}}^{\alpha_{1} \ldots \alpha_{k+1}}(x)$ are coefficients of tensors symmetric in the covariant indices $i_{1}, \ldots, i_{n}$. and symmetric in each block of $\alpha_{i}$ contravariant indices.

The spaces $\Omega\left(M, \mathscr{T}_{\text {poly }}\right)$ and $\Omega\left(M, \mathscr{D}_{\text {poly }}\right)$ have a formal fiberwise DGLA structure. Namely, the degree of an element in $\Omega\left(M, \mathscr{T}_{\text {poly }}\right)$ ( resp. $\Omega\left(M, \mathscr{D}_{\text {poly }}\right)$ ) is defined by the sum of the degree of the exterior form and the degree of the polyvector field (resp. the polydifferential operator), the bracket on $\Omega\left(M, \mathscr{T}_{\text {poly }}\right)$ is defined by $\left[\omega_{1} \otimes \mathscr{F}_{1}, \omega_{2} \otimes \mathscr{F}_{2}\right]_{S N}:=(-1)^{k_{1} q_{2}} \omega_{1} \wedge \omega_{2} \otimes\left[\mathscr{F}_{1}, \mathscr{F}_{2}\right]_{S N}$ for $\omega_{i}$ a $q_{i}$ form and $\mathscr{F}_{i}$ a section in $\mathscr{T}_{\text {poly }}^{k_{i}}$ and similarly for $\Omega\left(M, \mathscr{D}_{\text {poly }}\right)$ using the Gerstenhaber bracket. The differential on $\Omega\left(M, \mathscr{T}_{\text {poly }}\right)$ is 0 and the differential on $\Omega\left(M, \mathscr{D}_{\text {poly }}\right)$ is defined by $\partial:=\left[m_{p f}, .\right]_{G}$ where $m_{p f}$ is the fiberwize multiplication of formal power series in $y$ of $E$.

Definition 7 [7] The differential $D_{F}^{\mathscr{T}_{\text {poly }}}$ is defined on $\Omega\left(M, \mathscr{T}_{\text {poly }}\right)$ by

$$
D_{F}^{\mathscr{T}_{\text {poly }} \mathscr{F}}:=\nabla^{\mathscr{T}_{\text {poly }} \mathscr{F}}-\delta^{\mathscr{T}_{\text {poly }} \mathscr{F}}+[A, \mathscr{F}]_{S N}
$$

where $\nabla^{\mathscr{T}_{\text {poly }}} \mathscr{F}=d \mathscr{F}-\left[\sum_{i j k} d x^{i} \Gamma_{i j}^{k} y^{j} \partial_{y_{k}}, \mathscr{F}\right]_{S N}$ and where $\delta \mathscr{F}=\left[\sum_{i} d x^{i} \frac{\partial}{\partial y^{i}}, \mathscr{F}\right]_{S N}$. Similarly $D_{F}^{\mathscr{D} p o l y}$ is defined on $\Omega\left(M, \mathscr{D}_{\text {poly }}\right)$ by

$$
D_{F}^{\mathscr{D} \text { poly }} \mathscr{O}:=\nabla^{\mathscr{D}_{\text {poly }}} \mathscr{O}-\delta^{\mathscr{D} \text { poly }} \mathscr{O}+[A, \mathscr{O}]_{G}
$$


with $\nabla^{\mathscr{D}_{\text {poly }}}$ and $\delta^{\mathscr{D}_{\text {poly }}}$ defined as above with the Gerstenhaber bracket.

Again the cohomology is concentrated in degree 0 and a flat section $\mathscr{F} \in \mathscr{T}_{\text {poly }}$ or $\mathscr{O} \in \mathscr{D}_{\text {poly }}$ is determined by its terms $\mathscr{F}_{0}$ or $\mathscr{O}_{0}$ of order 0 in $y$; it is defined inductively by

$$
\mathscr{F}=\mathscr{F}_{0}+\delta^{-1}\left(\nabla^{\mathscr{T}_{\text {poly }}} \mathscr{F}+[A, \mathscr{F}]_{S N}\right) \quad \mathscr{O}=\mathscr{O}_{0}+\delta^{-1}\left(\nabla^{\mathscr{D} \text { poly }} \mathscr{O}+[A, \mathscr{O}]_{G}\right)
$$

On the other hand, if $s_{1} \ldots s_{k+1}$ are sections of $E$, we have for a $\mathscr{F} \in \Gamma\left(M, \mathscr{T}_{\text {poly }}\right)$ :

$$
D_{F}\left(\mathscr{F}\left(s_{1}, \ldots, s_{k+1}\right)\right)=\left(D_{F}^{\mathscr{T}_{\text {poly }}} \mathscr{F}\right)\left(s_{1}, \ldots, s_{k+1}\right)+\mathscr{F}\left(D_{F} s_{1}, \ldots, s_{k+1}\right)+\cdots+\mathscr{F}\left(s_{1}, \ldots, D_{F} s_{k+1}\right)
$$

and similarly for a $\mathscr{O} \in \Gamma\left(M, \mathscr{D}_{\text {poly }}\right)$.

Definition 8 [3] As in Cattaneo et al. we associate to a polyvector field $F \in T_{\text {poly }}^{k}(M)$ a section $F_{\phi} \in \Gamma\left(M, \mathscr{T}_{\text {poly }}\right)$ : for a point $x \in M$ one considers the Taylor expansion (infinite jet) $F_{\phi}(x ; y)$ at $y=0$ of the push-forward $\left(\exp _{x}\right)_{*}^{-1} F\left(\exp _{x} y\right)$. Clearly this definition implies that $X_{\phi}\left(f_{\phi}\right)=(X f)_{\phi}$ so that $F_{\phi}$ is uniquely determined by the fact that

$$
F_{\phi}\left(f_{\phi}^{1}, \ldots, f_{\phi}^{k+1}\right)=\left(F\left(f^{1}, \ldots, f^{k+1}\right)\right)_{\phi} \quad \forall f^{j} \in C^{\infty}(M) .
$$

Similarly we associate to a differential operator $O \in D_{\text {poly }}^{k}(M)$ a section $O_{\phi} \in \Gamma\left(M, \mathscr{D}_{\text {poly }}\right)$ determined by the fact that

$$
O_{\phi}\left(f_{\phi}^{1}, \ldots, f_{\phi}^{k+1}\right)=\left(O\left(f^{1}, \ldots, f^{k+1}\right)\right)_{\phi} \quad \forall f^{j} \in C^{\infty}(M) .
$$

Observe that $D_{F}^{\mathscr{T}_{\text {poly }}} F_{\phi}=0$ by 4.21 and 4.22 and similarly $D_{F}^{\mathscr{D} \text { poly }} O_{\phi}=0$, hence we have

Proposition 4 A section of $\mathscr{T}_{\text {poly }}$ is $D_{F}^{\mathscr{T}_{\text {poly }}}$-horizontal if and only if is a Taylor expansion of a polyvectorfield on $M$, i.e. iff it is of the form $F_{\phi}$ for some $F \in T_{\text {poly }}^{k}(M)$.

Similarly a section of $\mathscr{D}_{\text {poly }}$ is $D_{F}^{\mathscr{D} \text { poly }}$-horizontal if and only if is of the form $O_{\phi}$ for some $O \in D_{\text {poly }}^{k}(M)$. The terms in such a flat section are defined by tensors which are universal polynomials (involving concatenations of no-loop type) in the tensors defining the polyvectorfield (or differential operator) on $M$, the curvature tensor and their iterated covariant derivatives.

Observe also that a $D_{F}$ closed section $s \in \Omega^{q}(M, E)$ or $\mathscr{F} \in \Omega^{q}\left(M, \mathscr{T}_{\text {poly }}\right)$ or $\mathscr{O} \in \Omega^{q}\left(M, \mathscr{D}_{\text {poly }}\right)$ for $q \geq$ 1 is the boundary of a section defined by tensors which are given by universal polynomials (involving concatenations of no-loop type) in the tensors defining the section, the curvature tensor and their iterated covariant derivatives.

The isomorphisms obtained are isomorphisms of differential graded Lie algebras.

\section{Construction of a universal star products}

The construction of a star product on any Poisson manifold by Cattaneo, Felder and Tomassini proceeds as follows: one quantize the identification of the commutative algebra of smooth functions on $M$ with the algebra of flat sections of $E$ in the following way.

A deformed algebra stucture on $\Gamma(M, E)[[v]]$ is obtained through fiberwize quantization of the jet bundle using Kontsevich star product on $\mathbb{R}^{d}$. Precisely, one considers the fiberwize Poisson structure on $E$ defined by $\Lambda_{\phi}$ and the fiberwize Kontsevich star product on $\Gamma(M, E)[[v]]$ :

$$
\sigma *_{K}^{\Lambda_{\phi}} \tau=\sigma \tau+\sum_{n=1}^{\infty} \frac{v^{n}}{n !} U_{n}\left(\Lambda_{\phi}, \ldots, \Lambda_{\phi}\right)(\sigma, \tau)
$$


The operator $D_{X}^{G}$ is not a derivation of this deformed product; one constructs a flat connection $D$ which is a derivation of $*_{K}^{\Lambda_{\phi}}$. One defines first

$$
D_{X}^{1}=X+\sum_{j=0}^{\infty} \frac{v^{j}}{j !} U_{j+1}\left(\hat{X}, \Lambda_{\phi}, \ldots, \Lambda_{\phi}\right)
$$

where $\hat{X}:=D_{X}^{G}-X$ is a vertical vectorfield on $E$. The formality equations imply that $D_{X}^{1}$ is a derivation of the star product. Using the fact that $U_{1}(\xi)=\xi$ for any vector field $\xi$ and that, for $n \geq 2$, the maps $U_{n}\left(\xi, \alpha_{2}, \ldots, \alpha_{n}\right)=0$ if $\xi$ is a linear vector field, we see that

$$
D_{X}^{1}=D_{X}^{G}+\sum_{j=1}^{\infty} \frac{v^{j}}{j !} U_{j+1}\left(\hat{\hat{X}}, \Lambda_{\phi}, \ldots, \Lambda_{\phi}\right)
$$

where $\hat{X}=\sum_{i} X^{i}\left(-\partial_{y^{i}}+\sum_{k} A_{i}^{k}(x ; y) \partial_{y^{k}}\right)$ as defined in equation 4.10, so that it is given by universal polynomials (with no-loop type concatenatons) in the tensors defining $X$, the curvature tensor and their iterated derivatives. The connection $D^{1}$ is not flat so one deforms it by

$$
D:=D^{1}+[\gamma, \cdot]_{*_{K}}
$$

so that $D$ is flat. The 1 -form $\gamma$ is constructed inductively using the fact that the cohomology of $D^{G}$ vanishes.

The next point is to identify series of functions on $M$ with the algebra of flat sections of this quantized bundle of algebras to define the star product on $M$.

This is done by buildind a map $\rho: \Gamma(M, E)[[v]] \rightarrow \Gamma(M, E)[[v]]$ so that $\rho \circ D^{G}=D \circ \rho$. This map is again constructed by induction using the vanishing of the cohomology.

All these points show that the star product constructed in this way is universal.

Similarly, one can show that Dolgushev's formality is universal in the following sense. Dolgushev constructs a $L_{\infty}$ morphism from the differential graded Lie algebra of polyvectorfields on $M$ to the differential graded Lie algebra of polydifferential operators on $M$, giving its Taylor coefficients, i.e. a collection of maps $U_{j}^{D}$ associating to $j$ multivectorfields $F_{k}$ on $M$ a multidifferential operator $U_{j}^{D}\left(\alpha_{1}, \ldots, \alpha_{j}\right)$. The tensors defining this operator are given by universal polynomials (involving concatenations of no-loop type) in the tensors defining the $\alpha_{j}$ 's, the curvature tensor and their iterated cavariant derivatives.

Indeed this $L_{\infty}$-morphism is obtained in two steps from the fiberwize Kontsevich formality from $\Omega\left(M, \mathscr{T}_{\text {poly }}\right)$ to $\Omega\left(M, \mathscr{D}_{\text {poly }}\right)$ building first a twist which depends only on the curvature and its covariant derivatives, then building a contraction using the vanishing of the $D_{F}$ cohomology.

\section{References}

[1] F. Bayen, M. Flato, C. Frondsal, A. Lichnerowicz et D. Sternheimer, Deformation theory and quantization, Ann. Phys. 111 (1978) 61-110.

[2] M. Cahen, S. Gutt, Produits * sur les espaces affins symplectiques localement symétriques, C.R. Acad. Sc. Paris 297 (1983), série I, 417-420.

[3] A.S. Cattaneo, G.Felder and L.Tommassini, From local to global deformation quantization of Poisson manifolds, Duke Math. J. 115, 2 (2002) 359-352. 
[4] A.S. Cattaneo, G.Felder, On the globalization of Kontsevich's star product and the perturbative Poisson sigma model, hep-th/0111028.

[5] Simone Gutt, John Rawnsley, Equivalence of star products on a symplectic manifold: an introduction to Deligne's Cech cohomology classes", Journ. of Geom. and Phys.29 (1999) 347-392.

[6] Simone Gutt, John Rawnsley, Natural star products on symplectic manifolds and quantum moment maps, Lett. in Math. Phys. 66 (2003) 123-139.

[7] V.Dolgushev, Covariant and equivariant formality theorem, Adv. Math. 191, 1 (2005) 147177.

[8] B.V. Fedosov, a simple geometrical construction of deformation quantization. J. Diff. Geom. 40 (1994) 213-238

[9] M. Flato, A. Lichnerowicz and D. Sternheimer, Crochet de Moyal-Vey et quantification, C.R. Acad. Sc., t. 283, A (1976), 19

[10] M. Kontsevich, Deformation quantization of Poisson manifold, Lett. Math. Phys. 66, 3 (2003) 157-216. 